\title{
Co@N-doped Porous Carbon Derived from Co-MOFs as an Efficient Bifunctional Electrocatalysts for Full Water Splitting
}

\author{
Min Zhao ${ }^{1,2}$, Changjuan Hu ${ }^{l}$, Gaoliang Zhou ${ }^{l}$, Xinyi Shen ${ }^{l}$, Wei Tan ${ }^{l}$, Jianguo Lv ${ }^{1,2, *}$, Shengjie Sun ${ }^{l}$, \\ Yuxuan Ma ${ }^{l}$, Ying Wang ${ }^{l}$, Junjun Zhang ${ }^{l}$, Jin Yang ${ }^{l}$, Miao Zhang, ${ }^{2,3,}$, \\ Gang $\mathrm{He}^{3, *}$, Lei Yang ${ }^{4}$ \\ ${ }^{1}$ School of Physics and Materials Engineering, Hefei Normal University, Hefei 230601, China \\ ${ }^{2}$ Key Laboratory for Photoelectric Detection Science and Technology of Education Department of \\ Anhui Province, Hefei Normal University, Hefei 230601, China \\ ${ }^{3}$ School of Physics and Material Science, Anhui University, Hefei 230039, China \\ ${ }^{4}$ Department of Chemistry and Materials Engineering, Hefei University, Hefei 230601, China \\ *E-mail: 1vjg1@163.com (J. Lv), zhmiao@ahu.edu.cn (M. Zhang), hegang@ ahu.edu.cn (G. He).
}

doi: $10.20964 / 2021.02 .16$

Received: 14 September 2020 / Accepted: 22 November 2020 / Published: 31 December 2020

Co@N-doped porous carbon were prepared by a one-step pyrolysis method derived from Co-MOFs. The obtained Co@N-doped porous carbon have a large number of metallic Co nanoparticles and contain considerable mesopores. The result indicate that the Co@NC materials exhibit high degree of graphitization. The sample of Co-1.4@NC has the largest grain size of cobalt and high specific surface area of with massive mesopores. As a remarkable bifunctional electrocatalysts, Co-1.4@NC presents remarkable hydrogen evolution reaction (HER) and oxygen evolution reaction (OER) activities in alkaline medium for overall water splitting to produce $\mathrm{O}_{2}$ and $\mathrm{H}_{2}$. The result may be attributed to the rich catalytic active species as well as superior electrical conductivity, highsurface area and the porous structure. The cost-effective, highly active and stable Co@NC electrocatalysts have great potential for application in electrocatalytic overall water splitting and fuel cells.

Keyword: Co@N-doped porous carbon; Hifunctional electrocatalysts; Hydrothermal method; oxygen evolution reactions

\section{$\underline{\text { FULL TEXT }}$}

(C) 2021 The Authors. Published by ESG (www.electrochemsci.org). This article is an open access article distributed under the terms and conditions of the Creative Commons Attribution license (http://creativecommons.org/licenses/by/4.0/). 